\title{
Ethische Aspekte von Public Health
}

\author{
Alena Buyx $\cdot$ Stefan Huster
}

Online publiziert: 22. Juli 2010

(C) Springer-Verlag 2010

Der Einfluss von sozialen und anderen Umweltfaktoren auf die Gesundheit ist grundsätzlich seit langem bekannt. Zahlreiche epidemiologische Studien haben in den vergangenen Jahrzehnten an großen Kollektiven und über lange Zeiträume untersucht, wie die Gesundheit der Menschen mit ihrer Umgebung, ihrem Beruf, ihrem sozialen Umfeld und vielen weiteren Faktoren zusammenhängt. Aufbauend auf den frühen Studien dieser Art wie etwa der Framingham-Studie (begonnen 1948) oder der Whitehall-Studie (begonnen 1967) mehren sich die Hinweise auf die enorme Bedeutung sozio-ökonomischer und anderer Umwelt-Determinanten sowohl für den Gesundheitszustand einzelner Individuen als auch für die Gesundheit von Gruppen und ganzen Bevölkerungen. Parallel zu dieser Erkenntnis ist entsprechend die Bedeutung der mit diesen Determinanten befassten Disziplin gewachsen: New Public Health $^{1}$ erfreut sich zunehmender Aufmerksamkeit in Forschung und Politik. Letzteres ist nicht überraschend, geht es doch in Public Health um Fragestellungen und Probleme mit direkter Relevanz für verschiedene staatliche Institutionen.

Die Medizin- und Bioethik allerdings hat sich, so wird gegenwärtig vielfach beklagt, der Public Health-Thematik nur zögerlich zugewandt. Noch immer stehen im medizinethi-

Der Inhalt dieses Textes spiegelt nicht die Ansichten des Nuffield Council on Bioethics wider.

\author{
A. Buyx $(\square)$ \\ Nuffield Council on Bioethics, 28 Bedford Square, \\ WC1B 3JS London, Großbritannien \\ E-Mail: abuyx@nuffieldbioethics.org \\ S. Huster \\ Lehrstuhl für Staats- und Verwaltungsrecht mit besonderer Berücksichtigung des Sozialrechts, \\ Ruhr-Universität Bochum, Bochum, Deutschland
}

\footnotetext{
${ }^{1}$ Unter dem Sammelbegriff „Public Health“, der sich nur schwer ins Deutsche übertragen lässt, werden jene Fachbereiche versammelt, die sich der Aufdeckung, Analyse und Beeinflussung dieser Faktoren verschrieben haben, wie etwa die Epidemiologie sowie die Sozial- und die Arbeitsmedizin ([1], S. 11). Als „New Public Health" wird die Disziplin gelegentlich deswegen bezeichnet, weil sie sich innerhalb des letzten Jahrhunderts stark gewandelt hat - von einer Disziplin, die sich vornehmlich mit Hygiene und Seuchenbekämpfung befasst, hin zu einer Fachrichtung, die den Blick auf die vielfältigen Einflussfaktoren der Gesundheit richtet.
} 
schen „Mainstream“ solche Themen im Vordergrund, die entweder Leben, Leib und Leiden einzelner Individuen betreffen (Sterbehilfe, Transplantationsmedizin, Embryonenforschung usw.) oder die sich an der Entwicklung neuer und zum Teil spektakulärer Technologien entzünden (Chimärenbildung, Nanotechnologie etc.). Umgekehrt ist bisher ebenfalls recht wenig explizite Auseinandersetzung mit ethischen Aspekten des eigenen Arbeitsfeldes vonseiten der Public Health Community erfolgt. Angesichts dieser Situation ist in den letzten Jahren international und in ersten Ansätzen auch in Deutschland ein Diskurs zur Public Health-Ethik und -Politik entstanden. Es gilt, diesen weiter voranzutreiben und die Public Health-Thematik als ein wichtiges Arbeitsgebiet der Medizin- und Bioethik und verschiedener angrenzender Disziplinen zu verankern. Zu diesem Anliegen leistet dieses Themenheft einen Beitrag, indem es eine Reihe von Beiträgen verschiedener Experten versammelt, die unterschiedliche ethische Aspekte von Public Health beleuchten. Das Spektrum der bearbeiteten Themen reicht dabei von der Herausarbeitung normativer Grundlagen von Public Health bis hin zur Erörterung konkreter politischer Umsetzungsprobleme und Vorschläge für die Gesundheits- und Sozialpolitik. Den Anforderungen des Arbeitsfeldes Public Health entsprechend sind daher sowohl die empirischen Disziplinen als auch die normativen Fächer vertreten.

Im ersten Teil des Heftes widmen sich die Autoren wichtigen theoretischen und normativen Grundfragen der Public Health-Ethik und -Politik. Michael Quante wendet sich der Frage zu, wie eine Ethik aussehen könnte, die der modernen Public Health-Praxis zugrunde gelegt werden und in der Bewältigung der verschiedenen Herausforderungen Hilfe bieten könnte. Mit diesem Ziel vor Augen skizziert er einen normativen Rahmen, innerhalb dessen sich eine solche Ethik erfolgreich entwickeln lässt. Damit steckt er zugleich ein Forschungsprogramm für die Public Health-Ethik ab. Norbert W. Paul untersucht in seinem Beitrag die neuen Konzepte einer prädiktiv-präventiven Medizin und dabei insbesondere die einer Public Health-Genetik. In diesem Zusammenhang wendet er sich der Rolle verschiedener Grundwerte, wie etwa der Funktion der Medizin für moderne Gesellschaften, ebenso zu wie der Bedeutung sich wandelnder medizinischer Schlüsselbegriffe („Gesundheit“, „Krankheit"). Er weist dabei Public Health als ein Beispiel für die neuartige Zukunftsgewandtheit der Medizin aus und diskutiert den sich daraus ergebenden Rollenwandel.

Die beiden folgenden Beiträge schlagen den Bogen von der normativen Theorie hin zu den Problemen der Praxis und Politik von Public Health, indem sie sich mit dem zentralen Problem der Verantwortung für die Gesundheit beschäftigen. Georg Marckmann fragt, wie im Rahmen von Public Health, in dem es nicht um einzelne präventive oder kurative Maßnahmen am Individuum, sondern vornehmlich um Ansätze auf der Bevölkerungsebene geht, Verantwortung zugewiesen werden kann. Wie viel Verantwortung soll der Einzelne tragen und welche Einschränkungen der Entscheidungsautonomie des Einzelnen sind gerechtfertigt, um bestimmte Ziele von Public Health zu erreichen? Er entwickelt ethische Anforderungen an Public Health-Maßnahmen und kommt zu dem Schluss, dass nur eine prospektive Zuweisung von Verantwortung zu rechtfertigen sei. Dem gleichen Fragenkomplex wendet sich Alena Buyx zu. Sie stellt ein im angloamerikanischen Raum intensiv im Public HealthKontext verhandeltes Modell für die gerechtfertigte Berücksichtigung und Beeinflussung von Gesundheitsverhalten vor: den sogenannten libertären Paternalismus. Anhänger dieses Modells versuchen die umstrittenen Fragen der Verantwortungszuweisung zu umgehen. Buyx diskutiert den Ansatz kritisch hinsichtlich seiner Eignung als normatives Modell der modernen Public Health-Politik. Sie kommt zu dem Schluss, dass der libertäre Paternalismus in wichtiger Hinsicht theoretisch unterbestimmt ist, jedoch viele sinnvolle Impulse für die Public Health-Praxis liefern kann. 
Die Texte des zweiten Abschnitts widmen sich der Empirie und der Praxis der Public Health-Politik. Eine besondere Rolle spielen dabei die gesundheitliche Ungleichheit und die sich daraus ergebenden Gerechtigkeitsfragen, die auch angesichts knapper Ressourcen im Gesundheitswesen zunehmend an Bedeutung gewinnen. Einen weiteren Schwerpunkt bildet die Auseinandersetzung mit den juristischen und sozialpolitischen Herausforderungen, die sich daraus ergeben. Die Beiträge von Andreas Mielck und Siegfried Geyer stellen die Erkenntnisse der modernen Public Health-Forschung zum Zusammenhang von Sozialstatus und gesundheitlicher Ungleichheit differenziert dar und diskutieren verschiedene Modelle, diesen Zusammenhang zu erklären. Auf dieser Grundlage erörtern sie Handlungsoptionen zur Reduzierung von Ungleichheiten und sprechen auch die ethischen Kernfragen an. So ergeben sich fruchtbare Rückbezüge zu den Beiträgen im ersten Teil des Heftes. Oliver Rauprich knüpft an diese Diskussion an und fragt, ob und wann soziale Gesundheitsungleichheiten als ungerecht angesehen werden müssen. Er weist dabei auch darauf hin, dass der gesundheitspolitische Stellenwert von Public Health-Maßnahmen üblicherweise geringer ist als der Stellenwert der medizinischen Versorgung. Die Frage nach der Ungerechtigkeit sozialer Gesundheitsungleichheiten sei letztlich nur im Rahmen einer umfassenden Theorie der sozialen Gerechtigkeit zu beantworten. Der Beitrag von Volker H. Schmidt stellt aus der Perspektive der Theorie der Sozialpolitik die Frage, ob der hohe Anteil der öffentlichen Gesundheitsausgaben in Deutschland gerechtfertigt werden kann. Er spricht sich - auch und gerade mit Blick auf die soziale Gerechtigkeit - für eine Verschiebung der Prioritäten zugunsten des Bildungswesens aus und skizziert, wie unter dieser Prämisse das Gesundheitssystem reformiert werden müsste. Schließlich weist Stefan Huster darauf hin, dass der Grundsatz der Eigenverantwortung sowohl im Versorgungssystem als auch in der Public Health-Politik eine wichtige Rolle spielt. Er vergleicht die Diskussionen in beiden Bereichen und stellt ihren Zusammenhang dar.

Die Herausgeber danken den Autoren, den externen Gutachtern sowie dem Zentrum für medizinische Ethik e. V. an der Ruhr-Universität Bochum, das die Veröffentlichung dieses ungewöhnlich umfangreichen Themenhefts durch einen Druckkostenzuschuss ermöglicht hat.

\section{Literatur}

1. Schwartz FW, Badura B, Leidl R, Raspe H, Siegrist J (Hrsg) (2003) Das Public Health Buch. Urban und Fischer, München 